\title{
The Role of Right Anterior Insula and Salience Processing in Inhibitory Control
}

\author{
Ayda Ghahremani, ${ }^{1,2}$ Anuj Rastogi, ${ }^{1,2}$ and Susy Lam ${ }^{1,2}$ \\ ${ }^{1}$ Institute of Medical Science, Collaborative Program in Neuroscience, University of Toronto, Toronto, Ontario, M5S 1A8, Canada, and ${ }^{2}$ Toronto Western \\ Research Institute, Division of Brain, Imaging and Behavior-Systems Neuroscience, Toronto, Ontario, M5T 2S8, Canada
}

Review of Cai et al.

Understanding the functional organization of the brain in inhibitory control has been a challenge for cognitive neuroscientists for more than a decade. In its simplest form, such as outright inhibition in response to a Stop signal after the intended movement plan is initiated, high-level processing in the prefrontal cortex presumably generates a stopping command to cancel the plan before execution. Many studies investigating the functional organization of prefrontal regions during inhibition have agreed upon the right inferior frontal cortex (rIFC) as the most critical region for recruiting inhibition (Aron et al., 2014). In contrast, less is known about the role of the coactivated region in the right anterior insula (rAI), where activation was even more prominent (see metaanalysis by Swick et al., 2011).

Several views have emerged to explain the functions of rAI in cognitive task sets, including tasks that address inhibition. Some propose that the rAI maintains task rules, represented by its sustained activity throughout task performance (Dosenbach et al., 2008). Alternatively, rAI may maintain attention and focus during the task (Nelson et al., 2010). Neither of these accounts considers a specific role for rAI

Received Dec. 24, 2014; revised Jan. 14, 2015; accepted Jan. 20, 2015. We thank Drs. Robert Chen and Morris Moscovitch for insightful discussion. Correspondence should be addressed to Ayda Ghahremani, Toronto Western Hospital, 13-MP, Room 304, 399 Bathurst Street, Toronto, ON, M5T 258, Canada. E-mail: a.ghahremani@mail.utoronto.ca.

DOI:10.1523/JNEUROSCI.5239-14.2015

Copyright $\odot 2015$ the authors $\quad 0270-6474 / 15 / 353291-02 \$ 15.00 / 0$ in inhibitory control. A third, more compelling view, takes into account the well established role of $\mathrm{rAI}$ in interceptive awareness and processing of salient events (Craig, 2009; Uddin, 2015). According to this account, rAI, embedded in the salience network, detects salient events and coordinates other brain networks, such as central-executive networks, to guide behavior. The results of a recent study by Cai and colleagues (2014) are consistent with this account. Cai and colleagues (2014) reported not only that rAI plays a different role from rIFC in inhibitory control, but that it is embedded in the salience network that is functionally dissociated from rIFC and its interconnected network.

Distinguishing the roles of $\mathrm{rAI}$ and rIFC during inhibitory control is challenging because they are coactivated in many neuroimaging studies on inhibition. Cai and colleagues (2014) used novel multi-stepped quantitative approaches to reveal the functional segregation between rAI and rIFC. The study used multiple datasets: one resting state $(n=21)$, and two task-evoked ( $n=18, n=52)$ fMRI datasets from three independent cohorts of participants. The resting-state datasets were used to assess intrinsic activity of rAI and $\mathrm{rIFC}$ in the resting state, whereas the task-evoked datasets were used to assess specific recruitment of rAI and rIFC during inhibition. The core behavioral paradigm of the two task-evoked datasets was a Stop-Signal Task (SST). During the SST, subjects were instructed to press a button in response to a "Go" cue (on Go trials) and inhibit their response when a "Stop" cue (or Stop signal) occurred. Stop signals appeared randomly and at a variable delay after the Go cue (on Stop trials; $<30 \%$ of trials). This delay was adjusted so that stopping responses was successful at shorter delays (Successful Stop trials) and unsuccessful at longer delays (Unsuccessful Stop trials). Accordingly, this task fulfills the criteria to engage inhibitory control, by stopping movements that had already been initiated by preceding Go cues.

Cai and colleagues (2014) first conducted a meta-analysis (Activation Likelihood Estimation) of published neuroimaging studies on inhibitory control to determine voxels of interest (VOI) as the commonly activated region involving $\mathrm{rAI}$ and rIFC. Next, the authors used intrinsic activity and functional connectivity with the rest of the brain to cluster the VOI into rAI and rIFC. In addition, to segregate networks in which rAI and rIFC clusters were embedded, a seed-based approach was taken: a seed was selected in each area, and quantitative correlations between the seed and the rest of the brain generated functional connectivity maps. Functional connectivity represents synchronous fluctuations in the activity of brain regions defined as nodes, and it is increasingly used to identify brain networks in which a region is embedded. Furthermore, the contrasts between wholebrain functional connectivity maps associated with $\mathrm{rAI}$ and rIFC regions allowed the authors to determine their distinct networks. 
The results support the notion that rAI is part of the salience network, since it had strong functional connectivity with anterior cingulate cortex (ACC), a finding that is consistent with previous functional connectivity studies (for a review, see Uddin, 2015). In contrast, rIFC was functionally connected with medial frontal gyrus and supramarginal gyrus, key nodes of the lateral frontoparietal network, implicated in executive functions (Dosenbach et al., 2008) and called the central-executive network (CEN) (Uddin, 2015). Therefore, these findings suggest the existence of two separate networks for rIFC and rAI: CEN and the salience network, respectively. Importantly, using task temporal profiles, Cai and colleagues (2014) showed that the activity of rAI, but not rIFC, was greater on "Unsuccessful Stop" trials than on "Successful Stop" trials and was sensitive to the outcome of stopping. Conversely, the activity of rIFC, unlike rAI, was not sensitive to the outcome of stopping, but predicted the individual variability in the stopsignal reaction time as a measure of the stopping speed, a finding that is in line with the direct role of rIFC in the inhibitory control (Aron et al., 2014).

One important question arises from these results: What is the exact role of rAI and salience processing in inhibitory control? We suggest at least two non-mutually exclusive possibilities that explain the results of Cai and colleagues (2014): (1) rAI and salience networks may contribute to inhibitory control by detecting salient stopping events; and (2) rAI and salience networks may directly recruit inhibition by slowing down responses after salient events.

The first possibility is that rAI may integrate the salient information contained in the infrequent Stop signals that is further used by rIFC and CEN to recruit inhibition. Cai and colleagues (2014) found strong connectivity between rAI and ACC, the key nodes of the salience network, both during the resting state and during Stop trials in the task. This is consistent with prevailing models of insular functions describing the roles of $\mathrm{AI}$ in the context of the salience network. Moreover, these models posit that the salience network plays a central role in detecting behaviorally important cues and directing attention toward them (Uddin, 2015). In agreement with this notion, the greater activity of rAI on Unsuccessful Stop trials in the results of
Cai and colleagues (2014) is likely indicative of enhanced saliency of Stop signals on these trials. Since Stop signals on Unsuccessful Stop trials occur late after Go cues, when responses are close to execution, they are considered as behaviorally more salient. Together, the results of the current study support an indirect involvement of rAI and salience processing in inhibition by detecting the salient events in the task.

However, recent studies suggest an alternative hypothesis about the role of salience processing in inhibitory control. Much evidence indicates that salient or unexpected events by themselves can directly induce motor slowing, even if they are not Stop signals. For example, motor slowing was observed following unexpected error feedbacks in reward-prediction errors (Cavanagh et al., 2010) and following unexpected Go cues in reaction-time tasks, presumably by recruiting networks involved in inhibition (Wessel and Aron, 2013). Similarly, in the study by Cai and colleagues (2014), we speculate that salient Stop signals in the task not only attempt to inhibit the responses, but also attempt to slow responding to the preceding Go cues. Such motor slowing is especially important for Unsuccessful Stop trials, during which reaction times to the preceding Go cues are fastest, and stopping is less likely (Logan and Cowan, 1984). Therefore, the prominent activity of rAI on these trials in the findings of Cai and colleagues (2014) can be interpreted as effortful, but unsuccessful, force of the salience network to slow motor execution. More direct evidence concerning the recruitment of inhibition and motor slowing by salient events can be obtained using tasks that involve occasional salient or unexpected events other than Stop signals, such as a Novelty Task (Wessel and Aron, 2013). The Novelty Task is a reaction-time task in which subjects respond verbally or manually to an event that is either Regular (the most frequent event) or Novel (the salient event). This task can be combined with the quantitative techniques of Cai and colleagues (2014) to segregate clusters in rIFC and rAI and to elucidate the roles of their respective networks in motor slowing after Novel events. If slower responses to Novel events were associated with activations of rIFC and central-executive networks in addition to rAI and salience networks, this would more strongly support the direct contribution of salience processing to inhibitory control.

Together, the findings of Cai and colleagues (2014) relate at least two important research topics in cognitive neuroscience, namely salience processing and inhibitory control, by revealing the roles of rAI and salience networks in motor inhibition. In light of these findings, further research should pay closer attention to salience processing and prominent roles of rAI from the viewpoint of inhibitory control. These results open new avenues for future studies that might investigate the exact nature of salience processing in recruiting inhibition and its linkages with networks involved in motor slowing.

\section{References}

Aron AR, Robbins TW, Poldrack RA (2014) Inhibition and the right inferior frontal cortex: one decade on. Trends Cogn Sci 18:177-185. CrossRef Medline

Cai W, Ryali S, Chen T, Li CS, Menon V (2014) Dissociable roles of right inferior frontal cortex and anterior insula in inhibitory control: evidence from intrinsic and task-related functional parcellation, connectivity, and response profile analyses across multiple datasets. J Neurosci 34:14652-14667. CrossRef Medline

Cavanagh JF, Frank MJ, Klein TJ, Allen JJ (2010) Frontal theta links prediction errors to behavioral adaptation in reinforcement learning. Neuroimage 49:3198-3209. CrossRef Medline

Craig AD (2009) How do you feel-now? The anterior insula and human awareness. Nat Rev Neurosci 10:59-70. CrossRef Medline

Dosenbach NU, Fair DA, Cohen AL, Schlaggar BL, Petersen SE (2008) A dual-networks architecture of top-down control. Trends Cogn Sci 12:99-105. CrossRef Medline

Logan GD, Cowan WB (1984) On the ability to inhibit thought and action: a theory of an act of control. Psychol Rev 91:295-327. CrossRef

Nelson SM, Dosenbach NU, Cohen AL, Wheeler ME, Schlaggar BL, Petersen SE (2010) Role of the anterior insula in task-level control and focal attention. Brain Struct Funct 214:669680. CrossRef Medline

Swick D, Ashley V, Turken U (2011) Are the neural correlates of stopping and not going identical? Quantitative meta-analysis of two response inhibition tasks. Neuroimage 56: 1655-1665. CrossRef Medline

Uddin LQ (2015) Salience processing and insular cortical function and dysfunction. Nat Rev Neurosci 16:55-61. CrossRef Medline

Wessel JR, Aron AR (2013) Unexpected events induce motor slowing via a brain mechanism for action-stopping with global suppressive effects. J Neurosci 33:18481-18491. CrossRef Medline 